\title{
Chapter 5 \\ Social Meta-Learning: Learning How to Make \\ Use of Others as a Resource for Further \\ Learning
}

\author{
Jedediah W.P. Allen and Hande Ilgaz
}

\begin{abstract}
While there is general consensus that robust forms of social learning enable the possibility of human cultural evolution, the specific nature, origins, and development of such learning mechanisms remains an open issue. The current paper offers an action-based approach to the study of social learning in general and imitation learning in particular. From this action-based perspective, imitation itself undergoes learning and development and is modeled as an instance of social metalearning - children learning how to use others as a resource for further learning. This social meta-learning perspective is then applied empirically to an ongoing debate about the reason children imitate causally unnecessary actions while learning about a new artifact (i.e., over-imitate). Results suggest that children over-imitate because it is the nature of learning about social realities in which cultural artifacts are a central aspect.
\end{abstract}

Keywords Social learning • Over-imitation • Cultural affordances • Selfscaffolding • Social realities

\subsection{Theoretical Framework}

Neither humans nor robots can be preprogrammed to competently operate in real world physical environments: there is too much complexity and it cannot be anticipated beforehand what will be relevant for successful interaction (McCarthy \& Hayes, 1969). Instead, both humans and robots must be capable of learning. In the realm of social engagements, the issue of preprogramming is even more pronounced because the "objects" involved are ontologically emergent from the co-constituted activity of the participants (Bickhard, 2008). Accordingly, participation in a sociocultural world requires that humans and robots be equipped with the ability to learn

J.W.P. Allen $(\varangle) \bullet H$. Ilgaz

Psychology Department, Bilkent University, 06800, Ankara, Turkey

e-mail: jallen@bilkent.edu.tr; jedediahwpallen@gmail.com; hande.ilgaz@ bilkent.edu.tr 
from others (social learning), and, for human-like competence, to learn and develop their ability to learn from others (social meta-learning).

There is general consensus that participation in human culture and advances through cultural evolution have depended on the possibility of robust forms of social learning. Imitation is widely thought to be one of these robust forms of social learning but the specific nature, origins, and development of this form of learning garners far less agreement (Anisfeld, 2005; Jones, 2007; Gergely \& Csibra, 2005; Heyes, 2005; Tomasello, 1996). Further, the dominant theoretical frameworks in both animal and developmental research do not motivate a perspective in which imitation itself involves learning and development. In general, humans are not only capable of learning, but also, they are capable of learning to learn (meta-learning, Bickhard, 2005). With respect to social forms of meta-learning children are learning how to make use of others as a resource for subsequent (social/individual) learning and development. This perspective on imitation, as a form of social meta-learning, is motivated from within an alternative action-based framework for modeling development more broadly (Allen \& Bickhard, 2013a; Bickhard, 2009).

\subsubsection{An Action-Based Framework: Interactivism}

Theoretical models and assumptions about the nature and origins of representation, learning, and development involve sets of mutually constraining relations (Allen \& Bickhard, 2011a; Piaget, 1954). Using an action-based framework for modeling learning and development means being committed to (inter)action as the epistemic ground for the emergence of mind. For an action-based perspective, knowledge is constituted by interactive competence and representation is a matter of learning about relevant interactive possibilities.

Interactivism is a well-developed action-based framework that models the origins and ontology of representation, learning, and development for both the physical and social world (Bickhard, 1980, 2009, in preparation; Bickhard \& Terveen, 1995). The interactivist model of representation is ultimately grounded in the normative functioning of recursively self-maintaining systems that are far from thermodynamic equilibrium. Specifically, the minimal model of representation explicates how truth-value and aboutness are considered emergent properties of systems capable of successfully anticipating interaction potentialities that would be functional within the current situation (Bickhard, 2009). In this model, anticipation forms the core of representation.

\subsubsection{Object Representation}

The emergence of a more canonical form of representation has been demonstrated by borrowing from Piaget's own action-based model of object representation (Piaget, 1954). For interactivism, object representation is constituted by an invariant 
web of anticipations for potential interaction. Any point in this web of anticipations is reachable from any other point and the web remains invariant under a large class of other possible transformations (Bickhard, 2009). For example, an infant's interactive representation of a small wooden block is constituted by various possible visual scans, finger manipulations, and mouthing activities. These interactive possibilities remain available despite multiple other transformations such as visible and invisible displacement, occlusion, containment, and so on; however, if the block involves a transformation in which it is burned or crushed, the previously available interactive possibilities are gone.

\subsubsection{Social Agent Representation}

In contrast to physical objects, the interactive possibilities afforded by social agents are, largely, not perceptually available. Instead, your interactive characterization of a social agent depends on that other agent's interactive characterization of you. This creates an unbounded iteration of mutually dependent characterizations that must get resolved in order for the agents to successfully interact. Consequently, an accurate interactive characterization of a social agent is going to require knowing about the broader type of situation of which the two agents are major aspects. Therefore, successful interaction and coordination between social agents is made possible through the creation, invocation, and maintenance of mutually held interactive characterizations of the situation - i.e., social realities. In turn, social realities provide the basic ontology for learning about and navigating social life (Bickhard, 2008).

\subsubsection{Learning and Development}

From an action-based perspective, knowledge is constituted by interactive competence and, is therefore, inherently active, relational, and necessarily constructed through a variation and selection process (Bickhard, 2009). In contrast to nativism and empiricism, knowledge is not assumed to come from somewhere (genes or environment), nor is it assumed to be constituted by some sort of correspondence relationship between the mind and the world (Allen \& Bickhard, 2013b; Bickhard, 2009). Further, for an action-based approach, learning is not assumed to require a base set of representational primitives (Allen \& Bickhard, 2011b). Instead, knowledge is actively constructed through the creation of new internal organizations for the organism's possible functioning and internal organizations that enable successful interaction with the environment will be selected for. In short, learning is modeled as a variation and selection, emergent-constructivist, process (Allen \& Bickhard, 2011a).

For organisms that are capable of minimal forms of development, the constructive processes can be recursive in that current constructive processes can make 
use of prior constructive processes. ${ }^{1}$ In more complex organism, like humans, the constructive processes can themselves undergo learning. Such organisms will be capable of meta-recursive constructive processes - they will be cable of learning to learn (Bickhard, in preparation). From this perspective learning and development involve the same underlying dynamics but at different times scales. Accordingly, development will be constituted as the properties and constraints manifested by the historicities of learning - the ways in which prior learning influences future learning (Campbell \& Bickhard, 1992). For developmental research this will mean designing studies and interpreting results in the developmental context of what children have previously learned about the affordances of objects and social agents in different types of situations.

\subsubsection{Social Meta-Learning}

With respect to social forms of meta-learning children are learning how to make use of others as a resource for subsequent (social/individual) learning and development. In general, forms of social meta-learning are going to involve issues of the competence and the reliability of those others (Bickhard, in preparation). An early example of social meta-learning is when children develop attachment relationships that are adapted to specific people in their care-giving environment. Different attachment relationships emerge to enable children to maximize their use of the adult as an emotional resource. Children who develop a secure attachment relationship are able to consistently use the adult caregiver to help make global evaluations about situations and in that sense use the adult as a secure base from which to explore their environments. A later emerging form of social meta-learning is social referencing. Social referencing involves using an adult's emotional reaction to a particular object or event within a specific situation. This emotional reaction provides the child with a more specific evaluation about whether to proceed or withdraw from their ongoing activity.

Imitation is a powerful form of social meta-learning that remains particularly useful throughout the first 3-4 years of life. For imitation, children are learning about how to make use of adults as a resource for specific engagements with the physical, social, and cultural world. However, children must not only learn how to imitate another person's actions (solving the correspondence problem - Nehaniv \& Dautenhahn, 2002), but also: who to imitate, what to imitate, and when to imitate. Further, children must develop the ability to use imitation for different functions: learning, play, affiliation, trust, commitment, etc.

\footnotetext{
${ }^{1}$ Nativism and empiricism also have forms of recursive learning but both are restricted to the combinatorial spaces defined by their base sets of representational primitives and do not allow for emergent representation (Allen \& Bickhard, 2011a).
} 
Finally, social meta-learning has renewed importance once children develop sufficient language capabilities. "Trust" research has focused on the various cues that children learn to use as indicating the reliability of information coming from conflicting sources (Harris et al., 2012). In general, many of these cues are learned and reconciled with other cues at around age four. For example, at around age four, a person's past accuracy is recognized as a more relevant cue for predicting their future reliability over an affiliation cue like familiarity (Corriveau \& Harris, 2009).

Having outlined an action-based approach to the representation of social realities and the development of social meta-learning, we will apply this perspective to the empirical study of imitation learning. This application has two aspects: first, it proposes to replace the current focus on imitation fidelity with a perspective in which all imitation is selective and based on cognitive, motivational, and socialcognitive processes. Second, it proposes an alternative account for an ongoing debate within contemporary imitation research about the nature of over-imitation. The paper will conclude by suggesting that the proposed action-based framework is adequate to the task of grounding empirical research on imitation in a way that more closely resembles the richness of human culture and provides greater theoretical integration with other developmental phenomena as compared to extant frameworks. Such theoretical integration is crucial for robotics programs that want to develop systems capable of participating in the social realities of human culture. Further, only action-based frameworks have been able to naturalize representation and mind, at least at the level of theoretical explanation. In turn, such naturalism is a perquisite for the possible construction of artificial systems with the various emergent properties of relevance for developing social ontology.

\subsection{The Current Study}

\subsubsection{The Debate: Selective vs. Over-imitation}

Differences in the fidelity with which young children copy an adult demonstration has formed the basis for multiple debates and controversies regarding imitation learning in particular and social cognition more broadly (Call \& Carpenter, 2002; Lyons, 2009; Meltzoff, 1995; Whiten, 2006; Zentall, 2006). Demonstrations of low fidelity copying have often led to characterizations of imitation as being selective, flexible, and rationally applied to the specifics of a situation (Gergely, Bekkering, \& Kiraly, 2002; Southgate, Chevallier, \& Csibra, 2009; Williamson \& Markman, 2006). In contrast, demonstrations of high fidelity copying have led to characterizations of imitation as being direct, automatic, and tied by evolutionary selection pressures to the intentional actions of an adult model (Horner \& Whiten, 2005; Lyons, Young, \& Keil, 2007; McGuigan, Whiten, Flynn, \& Horner, 2007). These contrasting characterizations between selective and non-selective imitation have become particularly salient given several powerful demonstrations that young 
children will replicate causally unnecessary transformations on artifacts despite direct evidence that they are unnecessary. For example, children will replicate tapping on the top of a box as part of a sequence along with other causally necessary steps in their effort to open it. This phenomenon has been termed "over-imitation".

The current paper will address two intimately related issues: first, how to reconcile the conflicting characterizations between selective imitation and automatic over-imitation? Second, how to account for why children over-imitate? It is important to highlight that selective and over-imitation tend to manifest in different types of situations and at different ages. Specifically, selective imitation has tended to be demonstrated mostly during the second year of life and in situations that concern the selection of which actions to use to achieve some outcome on an object (e.g., selecting to use your head versus your hand to push a button to turn on a light). In contrast, over-imitation ${ }^{2}$ has been demonstrated most clearly after age three and in situations that concern the selection of which object-transformations to use without regard for the precise actions involved (e.g., removing a horizontal pin from a box without regard for whether the pin is pushed through or pulled out). Further, when the same task has been used in different studies with both younger and older age groups, there is a transition from more to less selectivity (Gergely, 2003; Huang, Heyes, \& Charman, 2006; McGuigan \& Whiten, 2009). The "developmental inversion" from more to less selectivity is difficult to reconcile if researchers are focused exclusively on cognitive aspects of children learning about objects because older children should be cognitively more competent about the working of artifacts not less. In short, why do more knowledgeable older children imitate object transformations that are "clearly" irrelevant while their less knowledgeable younger peers show more selectivity? This problem is further illustrated by the demonstration that adults over-imitate with even greater fidelity than young children (McGuigan, Makinson \& Whiten, 2011).

\subsubsection{Resolving the Debate: Cultural Affordances}

Uzgiris (1981) proposed a developmental transition in children's use of imitation that could provide a potential answer to the current developmental question. Uzgiris argued that the function of imitation transitions developmentally from serving a cognitive learning function to serving a predominantly social engagement function. Applied to the current debate, when young children display selectivity while learning something new in an imitation situation, they are manifesting aspects of their cognitive understanding. Further, when older children over-imitate, they

\footnotetext{
${ }^{2}$ Over-imitation is not just high fidelity imitation but also involves "clear" evidence that the high level of fidelity is causally unnecessary (Lyons et al., 2007).
} 
are manifesting an underlying social motivation for social engagement (Call \& Carpenter, 2009; Nielsen, 2006; Nielsen \& Blank, 2011; Nielsen, Simcock \& Jenkins, 2008).

In contrast to the social-motivational explanation, other researchers have argued that over-imitation manifests as a by-product of a useful cognitive-learning strategy in which copying all of the adults' intentional actions is typically adaptive (Lyons, 2009; Lyons, Damrosch, Lin, Deanna \& Keil, 2011; Lyons et al., 2007; Whiten, McGuigan, Marshall-Pescini, \& Hopper, 2009). These researchers assume that their experimental situations primarily involve an instrumental motivation to achieve a demonstrated outcome (e.g., to retrieve the contents in a novel container). ${ }^{3}$ Therefore, they interpret the imitation of causally irrelevant actions as a consequence of changes in the child's cognitive understanding about how an artifact actually functions. Accordingly, these researchers conclude that when children imitate what seem to be obviously irrelevant actions (e.g., tapping on the top of a container), it is because they believe that these steps are causally necessary for achieving the ultimate goal of retrieving the contents from within the apparatus.

From the current perspective, we agree with the social-motivational approach that children are motivated to interact socially with adults; however, success at such an interaction requires learning. That is, in order to successfully interact with adults, children must learn about the interactive affordances of the situation in which artifacts are going to be a central aspect. Therefore, we also agree with the cognitivelearning approach that children are learning about the artifact; however, the meaning of what they are learning is intrinsically tied to the broader social interaction with the adult. Consequently, children's imitative responses will have less to do with their understanding of the causal necessity of different object transformations and more to do with the culturally relevant object affordances that children presume to be available for successful interaction in the situation.

For an example, consider learning how to play billiards through imitation. A model demonstrates how the pool cue is used to hit the cue ball and knock other balls into the holes. If an individual were to use all of his knowledge about causal necessity he would conclude that it would be more efficient to use his hands rather than the pool cue. After all, the goal is to get the balls into the holes. However, we would not consider this selective imitation (or even emulation). Instead, we would interpret this behavior as an indication that this individual is unwilling to participate in the social reality we call "billiards". In general, social realities are constituted by individuals who possess a shared interactive characterization of the situation (Bickhard, 2008). Learning about these shared characterizations suggests a sense in which imitation is able to contribute to the sharing of culture more broadly through the learning of new social realities. Importantly, imitation situations are

\footnotetext{
${ }^{3}$ Lyons and colleagues $(2007,2011)$ have attempted to specifically test this assumption with different conditions from two sets of studies as part of their effort to rule out the alternative social motivational interpretation for over-imitation in general; however, the current study suggests that these alternative control conditions have been inadequate.
} 
somewhat unique in that they also constitute a class of social reality themselves. Thus, as a social reality, imitation involves learning and development, while also being used to teach children about new social realities. Consequently, imitation serves as a powerful social bootstrapping mechanism for learning about culturally relevant social realities.

The above example serves to illustrate the basic point about the interactive nature of social realities involving cultural artifacts; however, it also highlights the limits of trying to define different forms of social learning solely in terms of the fidelity of the match between model and imitator. The very distinction between low fidelity selective imitation and high fidelity over-imitation derives from a theoretical perspective in which imitation is defined by a somewhat arbitrary degree of abstraction - how perceptually similar must the child's actions be in order to qualify as selective or not (McGuigan \& Whiten, 2009)? Imitation always involves some degree of abstraction away from the modeled demonstration, ${ }^{4}$ therefore, imitation always involves some degree of selectivity about what does and does not get copied. From the perspective being advocated in the current study, all imitation activity is selective and that selectivity always involves both cognitive and motivational aspects that are themselves relative to the child's understanding of the broader type of situation.

\subsubsection{Imitation as a Type of Social Situation}

To date, little of the research on over-imitation has directly examined the influence of the broader type of situation on the fidelity with which preschool children copy an adult model. Part of the power of the original study by Gergely et al. (2002) was to highlight the essential role that children's understanding of the broader type of situation has on how they interpret the activity from an adult demonstration. Despite some controversy regarding particular findings (Paulus, Hunnius, Vissers, \& Bekkering, 2011), one of the central lessons from the research program of Gergely and his colleagues is that children's selection of what to imitate depends on what they presume to be new or relevant about the adult demonstration (Csibra \& Gergely, 2009; Kiraly, Csibra, \& Gergely, 2013). While these presumptions of relevance may improve and change with the development of children's learning about artifacts and agents ("rational" constraints being a part of this learning), they will always be relative to what the child understands about the broader type of social situation as well.

In general, children have an extensive developmental history of experiences interacting with different types of objects and with adults in different types of situations. Accordingly, they have learned to apply different sets of general

\footnotetext{
${ }^{4}$ At bare minimum, it requires abstraction away from the actions of the model to those of the imitator.
} 
expectations about how to interact depending on the type of situation that has been indicated (pedagogical, imitation, play, helping, etc). For imitation, these general expectations involve making a variety of presumptions about what aspects of the current demonstration are potentially relevant. These presumptions are often tentative and always fallible but children have learned that some things are much more likely than others in any given situation (Schulz, Hooppell, \& Jenkins, 2008). Further, what is potentially relevant in a given situation depends on one's purposes.

Children come to learn that there are different types of imitation situations that may emphasize the different functions that imitation can serve. The two most common functions in the literature concern the "cognitive-learning" and "social-motivational" functions. However, the cognitive-learning function can be extended to learning about cultural artifacts in the context of learning about social realities. Learning about social realities constitutes the more general phenomenon that we argue is captured by standard over-imitation studies. Therefore, the current study sought to explore how the presumptions that children make during an adult demonstration of operating a novel artifact depends on their previously learned knowledge about artifacts as physical/cultural objects (cognitive aspects), current purposes (motivational aspects), and how both of them are relative to the child's knowledge about the broader type of social situation (social-cognitive aspects).

The general procedure for the current study was as follows: During the demonstration phase of the experiment (i.e., the imitation situation), all children were given four demonstration and response trials for how to open two identical boxes. The demonstration involved five distinct actions but only two of them were causally relevant to open the box. The demonstration phase was followed by the cooperation phase (i.e., the helping situation) in which children were given the opportunity to use what they had learned about opening the boxes to help an adult confederate find a toy that was hidden inside one of them. However, the boxes were surreptitiously locked and would only open if all five target-actions were performed.

\subsubsection{Rationale for the Hypotheses}

\subsubsection{Hypothesis 1}

Although a developmental trend from more to less selective imitation seems clear when looking across the literature as a whole, there is little direct evidence for a specific developmental increase in over-imitation for children in the preschool years (Lyons et al., 2007; McGuigan et al., 2007). In the current study, it was hypothesized that over-imitation would increase in age such that 3-year-olds would copy irrelevant actions the least, followed by 4 -year-olds, with 5-year-olds copying irrelevant actions the most. 


\subsubsection{Hypothesis 2}

Similar to the general paradigms of other over-imitation research (Horner \& Whiten, 2005; Lyons et al., 2007; McGuigan et al., 2007), children were invited to learn about an unfamiliar artifact through adult modeling. Specifically, the adult experimenter demonstrated a sequence of five target actions with a wooden box that resulted in the box being opened. Only two of these interactions were causally relevant to open the box and the other three were increasingly less plausible as being potentially relevant (i.e., rotating a non-functional lever, pushing a large button, tapping on the box three times). It was hypothesized that children would display differential copying of the five target actions in accordance with their prior experience such that the two causally relevant steps (removing the pin \& pulling the knob) were copied most.

\subsubsection{Hypothesis 3}

The cognitive-learning perspective on over-imitation assumes that children imitate the demonstrator with an instrumental motivation to open a novel artifact (Horner \& Whiten, 2005; Lyons et al., 2007, 2011). Thus, the steps children choose to copy are assumed to be instrumental for the goal of opening that artifact; therefore, the steps that they choose are interpreted as an indication of their understanding of how the artifact actually functions. However, the current study challenges the claim that canonical over-imitation situations primarily involve an instrumental motivation and, thus, the conclusion that children's copying behavior is an indication of what they understand to be causally necessary about opening the artifact.

Instead, because children have extensive experience with imitation type situations, they have a set of expectations about how to interact with the adult and are motivated to copy the adult's actions as much as they are motivated to open the artifact. Therefore, the current study compared children's actions on the box during a canonical over-imitation situation with their actions during a helping situation. The idea here is that a helping situation primarily involves an instrumental motivation because opening the box becomes decidedly instrumental for the broader goal of helping the adult. That is, the current study sought to manipulate children's underlying motivation by changing the purpose of the broader social situation - from learning through imitation to using what they learned about the box (in the canonical imitation situation) to accomplish their current goal of helping the adult. Therefore, it was hypothesized that over-imitation depends on the broader social situation such that children would be less likely to over-imitate in the helping situation relative to the canonical imitation situation. 


\subsubsection{Hypothesis 4}

There was also a more subtle motivational manipulation that attempted to change the focus from within the imitation situation itself. There is some evidence that changes in the verbal framing of a demonstration can have consequences for children's understanding of its purpose (Kiraly, 2009; Herrmann, Legare, Harris, $\&$ Whitehouse, 2013). Therefore, by reframing the verbal instructions during the demonstration phase, the current study sought to highlight the problem-solving goal of opening the box in one condition while highlighting the goal of playing in the other condition. It was hypothesized that verbal framing would alter children's imitation behavior such that irrelevant actions would be copied more in the play condition and relevant actions would be copied more in the problem-solving condition.

In sum, the primary purpose of the current study was to explore the implications of characterizing imitation activity as a consequence of both the cognitive and motivational aspects involved with resolving uncertainty (via social learning) in different types of situations. Doing so involves trying to answer two intimately related issues in the literature: first, how to reconcile the apparent conflict between selective imitation and automatic over-imitation? Second, how to account for the underlying reason why children over-imitate?

\subsubsection{Methods}

\subsubsection{Participants}

Seventy-four, predominantly white, middle class children (47 male, 27 female) who were 3-5 years old (3-year-olds, $N=27, M=37$ months, $\mathrm{SD}=1$; 4-year-olds, $N=21, M=49$ months, $S D=1$; 5-year-olds, $N=20, M=61$ months, $S D=1$ ) participated in the study. Children were recruited through published birth announcements and from the university daycare. Of the seventy-four children, six children were excluded from coding and analyses ( 2 for being uncooperative and 4 for not wanting to take a turn opening the boxes).

\subsubsection{Apparatus}

Materials included some stuffed animals, two punch buttons, and two wooden boxes $(30 \mathrm{~cm} \times 30 \mathrm{~cm} \times 20 \mathrm{~cm})$ differing only in color. Each box had a drawer with a $\mathrm{knob} / \mathrm{handle}$ on the front to open it. The drawer could be locked by a pin in the top of the box with a non-functional rotating lever attached behind the pin and rotated 180 degrees. Finally, a screen facade placed on top of the box and over the focal point of the lever also hid a surreptitious locking device on the side of the box. 


\subsubsection{Design}

The design can be summarized as follows: 3 (age group: 3-, 4-, \& 5-year-olds; between subjects) $\times 2$ (verbal framing: play vs. problem-solving; between subjects) $\times 5$ (demonstration: step1, step2, step3, step4, step5; within-subjects) $\times 2$ (situation type: imitation vs. helping; within-subjects).

\subsubsection{Procedure}

The procedure involved a variation on the active helping paradigm (Buttelmann, Carpenter \& Tomasello, 2009). While this procedure was not originally used to study imitation it was ideally suited for the current purposes in that it involved a demonstration phase on how to open two unfamiliar boxes followed by a cooperation phase where children made use of their new found knowledge in order to help the adult.

After a warm-up session with two experimenters (E1 \& E2), the demonstration phase began and the child was seated on a cushion that was equidistant (approximately $2 \mathrm{~m}$ ) from the two boxes that were themselves about $1 \mathrm{~m}$ apart. The two punch-buttons were placed approximately $30 \mathrm{~cm}$ away from the inside edge of the boxes, the drawers were open, and the locking pin for each box was on the floor directly in front of them (approximately $10 \mathrm{~cm}$ ). E1 was seated next to the child while E2 was seated between the two boxes facing the child and E1. After exploring the boxes briefly, E2 left the room in search of their favorite toy while E1 demonstrated to the child how the boxes could be opened with two response trials for each box.

Children were randomly assigned to one of two conditions that manipulated the verbal framing of the demonstration. Specifically, the experimenter introduced the task either as an opportunity to learn how to play with the boxes "Let me show you how to play with the boxes" (play condition) or as an opportunity to learn how to open the boxes "Let me show you how to open the boxes" (problem-solving condition). E1 provided two demonstrations on the first box (counterbalanced) before the child was given the first of their two turns on that box. E1 provided one additional demonstration on the second box before the child was given the first of their two turns on that box. No reinforcement was given while children took their turns with the boxes. Finally, between all turns, E1 asked children to return to the pillow, to cover their eyes, and obstructed their view in order to guarantee that children did not see how the boxes were reset.

A 5-step procedure that was identical for both boxes was demonstrated to children: Two of these steps were causally relevant and three were causally irrelevant. The two relevant steps included step 3: pulling a "locking" pin out of a hole; and step 5: pulling on a knob/handle to open the drawer. The three irrelevant steps were designed to be differentially plausible. Of these, step 2 was intended to be the most implausible and involved rotating a lever on the top of the box from right to left $180^{\circ}$. Step 1 involved a punch button that was pushed once and step 3 
involved tapping the "locking" pin on the edge of the box three times. Tapping on the box was thought to be less plausible than pushing the button but previous research has suggested that physical contact may mediate functional inferences (Lyons et al., 2007).

After the demonstration phase with E1, E2 returned to the room with their favorite toy and engaged the child in play. After $90 \mathrm{~s}$, E2 placed the toy in one of the boxes (counterbalanced) and left the room. Next, E1 invited the child to play a trick on E2 by moving the toy to the other box and ensured that both boxes were reset (locked). At this point, the cooperation phase began. The transition in phases from demonstration (i.e., imitation situation) to cooperation (i.e., helping situation) constituted the within-subjects manipulation of motivation. Upon re-entering the room, E2 attempted to open the now empty box where they had previously left their toy. Unable to open the box, E2 sat in a position centered between the two boxes, faced the child and looked "disappointed", "puzzled", and "resigned" (Buttelmann et al., 2009). Next, the response period began in which the child was able to help E2 open either of the boxes; however, the boxes were surreptitiously locked and would only open if the child performed all five target-actions in the correct sequence. If children did not spontaneously try to help E2, then they were prompted to do so but were not given any cues about how to open the box (e.g., E1: "Go on, you can help him/her" and/or E2: "Can you help me" and/or E1: "We can go together and help him/her").

The procedure can be summarized as follows: During the demonstration phase of the experiment (i.e., the imitation situation), all children were given four demonstration and response trials for how to open the two identical boxes (evenly and randomly split between play and problem-solving conditions). Following this was the cooperation phase (i.e., the helping situation) in which children were given an opportunity to use what they learned about opening the boxes to help an adult confederate. However, the boxes were surreptitiously locked and would only open if all five target-actions were performed.

\subsubsection{Coding and Reliability}

All of the analyses concerning social-learning aspects of the study were based on the first five actions that children performed on the boxes averaged across the four training trials ( 2 for each box); however, this did not include consecutive repeats (e.g., steps $1,1,2,3$ would be coded as 1,2,3 but 1,2,1 would be coded as 1,2,1). The resulting target action (TA) score was based on how many of the 5 target actions that children performed within the first five actions that they performed $(\max =5,1$ for each of the distinct target actions, e.g., 1,2,1 would have two TAs whereas 1,2,3 would have three TAs). For hypothesis 2 , concerning the influence of children's prior artifact knowledge, a total TA score was calculated on the bases of the total number of times that children produced any of the five distinct target actions throughout the demonstration phase; again, consecutive repeats were not included. There was no maximum because children were not forced to stop after their first five actions. 
All of the data was recoded by the collective efforts of three observers who independently viewed the videos and were unaware of the specific hypotheses of the experiment. There was $91 \%$ agreement between the original and recoded data. Discrepancies were settled by reviewing the video recordings. It was typically clear that one of the coders had made a mistake rather than there being any disagreement on the status of an ambiguous action. For those few cases where the action was ambiguous, a third coder settled the disagreement.

\subsubsection{Results}

\subsubsection{Hypothesis 1: Did Over-imitation Increase with Age?}

In order to test hypothesis 1, a multivariate analysis of variance (MANOVA) was conducted to assess whether there were differences in the number of relevant and irrelevant target actions performed across age groups (3-, 4-, \& 5-year-olds) and verbal framing conditions (play vs. problem-solving). Results indicated that imitation of relevant actions did not differ across the three age groups whereas imitation of irrelevant actions increased with age $\left(F(2,62)=10.25, p<.001, M_{3}=\right.$ $\left.1.69, S D=.79 ; M_{4}=2.35, S D=.51 ; M_{5}=2.48, S D=.51\right)$. Post hoc analyses (LSD) revealed significant differences between 3- and 4-year-olds $(p<.001)$ and 3 - and 5-year-olds ( $p<.001$ ), but not between the two older groups (see Fig. 5.1).

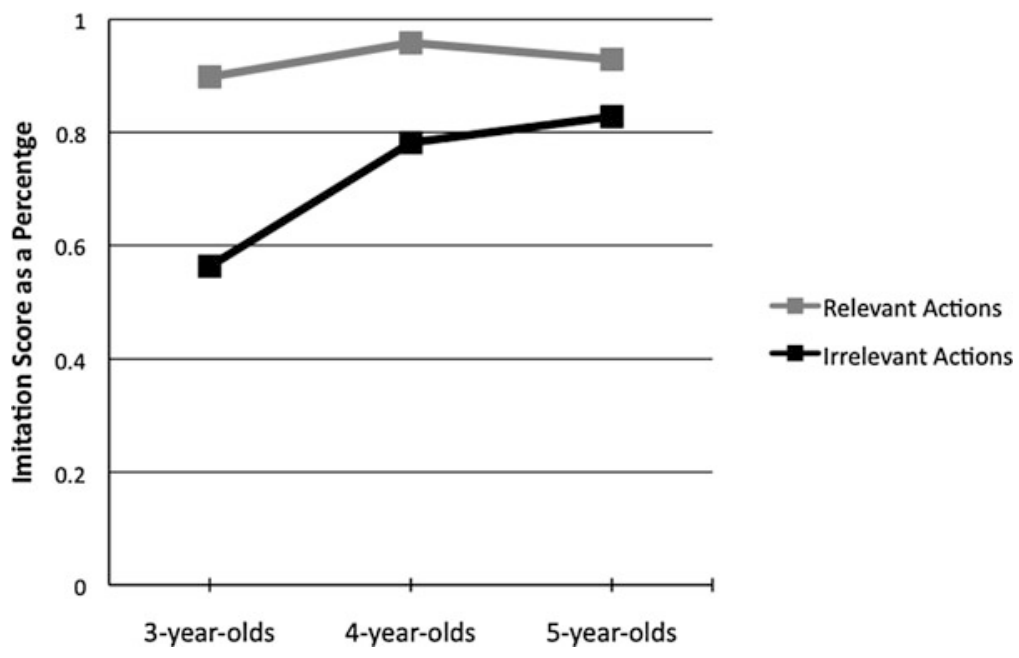

Fig. 5.1 Imitation scores for relevant and irrelevant target actions for 3-, 4-, and 5-year-olds. Children's imitation score is given in a percentage for clarity of comparison because there were only two relevant actions possible while there were three irrelevant actions possible. Post hoc analyses indicated that for irrelevant actions only, 4- and 5-year-old children differed significantly from 3 -year-olds ( $p<.001$ for both) but not from each other 


\subsubsection{Hypothesis 2: Were Children Sensitive to the Difference Between Relevant and Irrelevant Actions?}

To test hypothesis 2, a mixed model analysis of variance (ANOVA) was conducted to assess whether there were differences in the total number of times each of the five target actions was performed at any point in the demonstration phase with age (3-, 4-, \& 5-year-olds) and verbal framing (play or problem-solving) as between subjects variables. Although the assumptions of sphericity were violated, Greenhouse-Geisser corrections did not alter the statistical decision for any of the effects. The overall analysis indicated a significant effect of $\operatorname{step}(F(3.22,200.45)=$ $17.90, p<.001)$. Main effects for age and for verbal framing were marginally significant $\left(F_{\text {Age }}(2,62)=2.54, p=.087, \eta^{2}=.076 ; F_{\text {Framing }}(1,62)=3.25, p=\right.$ $.076, \eta^{2}=.050$ ). Specifically, 3-year-olds copied fewer overall target actions than 4- and 5-year-olds and children in the problem-solving condition copied more overall target actions than those in the play condition.

None of the possible interactions approached significance. In particular, there was not a significant interaction between step and age $(F(6.47,200.45)=1.50, p=$ n.s.) indicating that the relative differences between overall imitation of the five steps did not differ across the three age groups. Finally, follow-up analyses indicated that children preferentially copied those actions that were more likely to be relevant for actually opening the box. That is, all age groups tended to imitate the two relevant actions most followed by the irrelevant actions (see Table 5.1). Withinsubject contrasts confirmed that children differentiated between the relevant and irrelevant target actions. Specifically, step 5 was significantly different from steps 1, 2 , and $4(p<.001)$ but not step 3 .

\subsubsection{Hypothesis 3: Does Over-imitation Depend on the Broader Type of Situation?}

This hypothesis was being tested against an alternative hypothesis that children over-imitate (imitate irrelevant actions) because they have incorporated those actions into their "causal belief structure" for how to open the box independently of the type of situation (Lyons et al., 2007, 2011). To test these alternative hypotheses, children's imitation activity on their last trial of the demonstration phase (imitation

Table 5.1 Average number of times that children produced each of the five distinct target actions at any point throughout the imitation phase

\begin{tabular}{l|l|l|l}
\hline & $\mathrm{M}$ & $\mathrm{SD}$ & Relevant Action \\
\hline Step 1 (button press) & 3.29 & 1.37 & \\
\hline Step 2 (lever turn) & 2.82 & 1.67 & \\
\hline Step 3 (pin pull) & 4.04 & 1.27 & $*$ \\
\hline Step 4 (tap 3 times) & 2.49 & 1.73 & \\
\hline Step 5 (pull knob) & 4.13 & 1.64 & $*$ \\
\hline
\end{tabular}

Note. Step 5 differed significantly from steps $1,2 \& 4$ $(p<.001)$ but not step 3. The * indicates that the step was a relevant action 


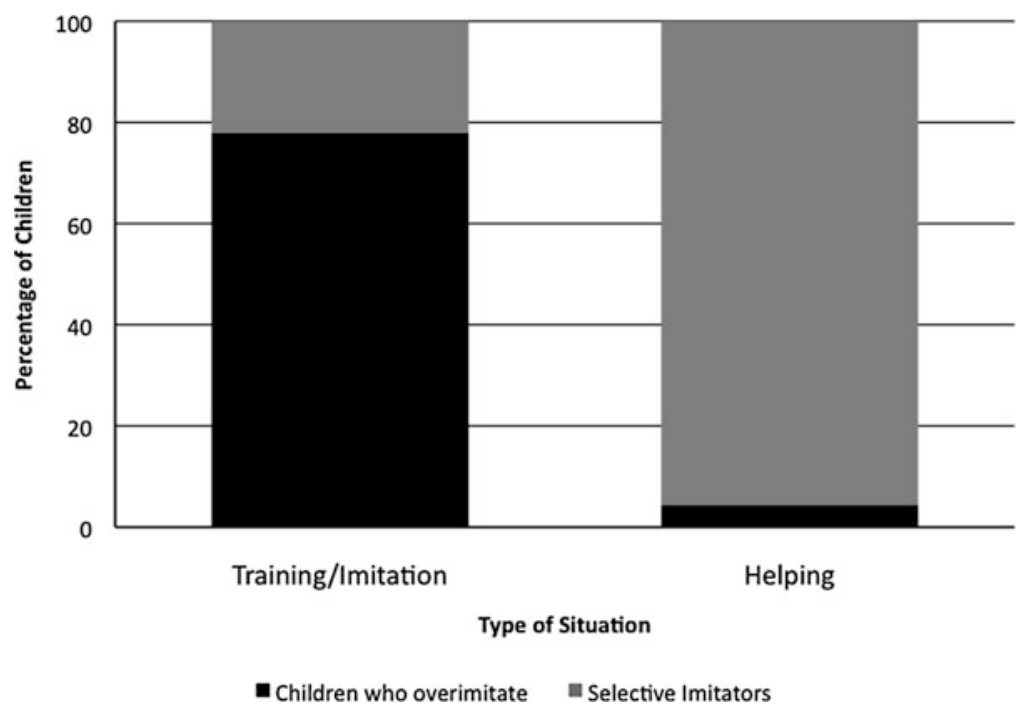

Fig. 5.2 Percentage of children who over-imitated in the imitation situation versus the helping situation of the experiment $\left(\chi^{2}=72.43, p<.001\right)$

situation) was compared to their first trial in the cooperation phase (helping situation). In both cases their actions were only coded until they attempted to open the box by pulling on the knob (i.e., step 5). Four children did not want to respond during the helping phase of the experiment and were not included in the analysis of that phase. Children were categorized as over-imitators if they performed two or more of the irrelevant actions. A Chi-Square analysis indicated that significantly more children over-imitated in the imitation situation $(N=53 / 68=78 \%)$ than in the helping situation $\left(N=3 / 64=5 \% ; \chi^{2}=72.43, p<.001\right.$, see Fig. 5.2).

Follow up analyses indicated that $3-, 4-$, and 5-year-olds all differed significantly in their over-imitation behavior across the two situations $(p<.001)$. That is, all three age groups demonstrated a significant decline in over-imitation from the imitation to the helping situation (see Fig. 5.3). However, 3-year-olds differed significantly from 4- \& 5-year-olds in terms of the proportion of children who overimitated in the imitation situation versus the helping situation $\left(\chi^{2}=13.17, p=\right.$ $.001)$. While just over half $(56 \%)$ of 3 -year-olds over-imitated in the imitation situation, $90 \%$ of 4 -year-olds and $95 \%$ of 5-year-olds over-imitated in the imitation situation (see Fig. 5.3).

Although children aged 3-5 and younger should not have a problem remembering a 5-step sequence (Bauer \& Fivush, 1992; McGuigan \& Whiten, 2009), we ensured that children who did not over-imitate in the helping situation could demonstrate that they had not forgotten the irrelevant steps. After failing to open the box using the two causally relevant actions (it was surreptitiously locked), $75 \%$ of children made spontaneous use of the irrelevant actions. 


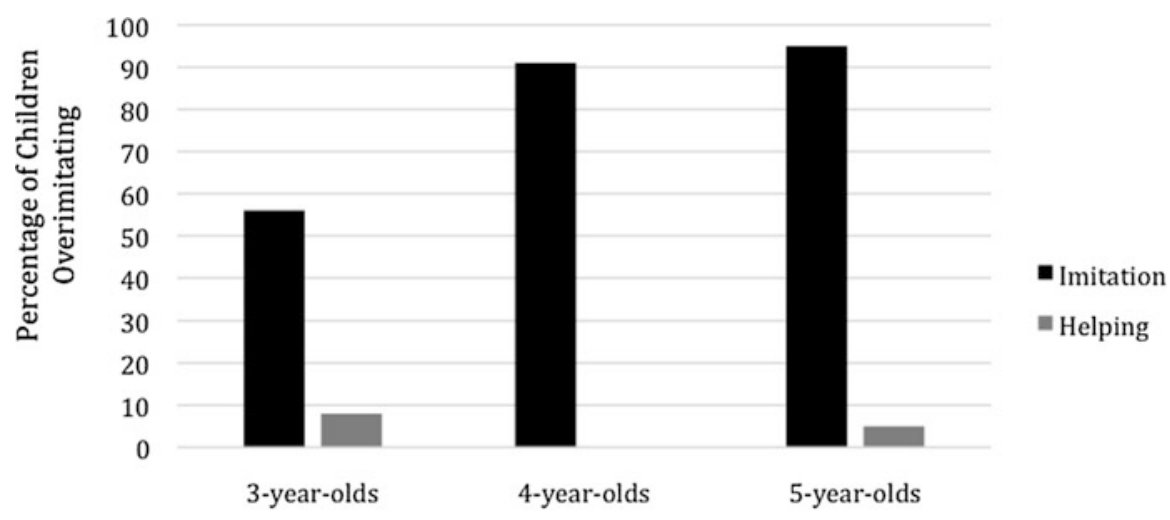

Type of Situation by Age

Fig. 5.3 Percentage of children who over-imitated in the imitation situation versus the helping situation divided by age group

\subsubsection{Hypothesis 4: Does Verbal Framing Effect Imitation Response?}

The analysis for the age hypothesis (hypothesis 1) assessed whether there were differences in the number of relevant and irrelevant target actions performed across age groups and verbal framing conditions (play vs. problem-solving). Results for the verbal framing variable revealed a marginal main effect for the imitation of relevant $(F(1,62)=3.87, p=.054)$ but not irrelevant $(F(1,62)=.57, p=n . s$.$) target$ actions. Specifically, children in the problem-solving condition imitated the relevant actions more often $(M=1.91, S D=.16)$ than did children in the play condition $(M=1.78, S D=.34)$.

\subsubsection{Summary}

First, over-imitation increased with age. Second, children differentially imitated the relevant versus irrelevant actions. Third, what actions children copied changed with the type of situation such that they over-imitated significantly less in the helping situation. Fourth, verbal framing had a moderately significant effect for relevant but not irrelevant actions.

\subsubsection{Discussion}

The purpose of the current study was to explore two intimately related issues in the literature: first, how to reconcile the apparent conflict between selective imitation and automatic over-imitation? Second, how to account for the underlying reason why children over-imitate? 
Three- to 5-year-old children come to an experimental imitation situation with a rich developmental history of learning and experience interacting with both physical objects and social persons. Social interactions inherently involve a broader type of situation and when these situations involve novel aspects there will be uncertainty about how to proceed; however, such uncertainty will not be absolute. That is, in a social situation involving novel aspects, how children proceed will be heuristically guided by their prior knowledge and learning about objects and people in different types of situations. Natural Pedagogy has aptly demonstrated that canonical imitation experiments have a number of social-communicative markers that orient children's understanding about the type of situation that they are involved with (Csibra \& Gergely, 2009). This orienting helps them to interpret the purpose of the situation and facilitates how to act "successfully" when given their turn. Accordingly, in a canonical imitation situation involving a relatively novel artifact, selecting what to copy from an adult demonstration will involve three interrelated aspects: social-cognitive, cognitive and motivational.

\subsubsection{Beyond Artifacts and Agents: Social-Cognitive and Motivational Aspects}

A major result from the current study (i.e., hypothesis 3) was the large decrease in the number of children who over-imitated in the helping situation relative to the imitation situation. Nearly $80 \%$ of children in the demonstration phase overimitated, while this number dropped to less than $5 \%$ in the cooperation phase. This finding is in stark contrast to Lyons and colleagues' $(2007,2011)$ efforts to demonstrate that over-imitation involves a stable distortion to the child's causalbelief structure about how the artifact operates.

\section{Cognitive-Learning Perspective}

While the current results are consistent with the conclusion that children are learning something about the box during the imitation situation, we disagree that children are learning about the "causal structure" of the box. ${ }^{5}$ Instead, it is a type of interactive or user knowledge similar to the type of learning typical of most adults for how to operate a car (i.e., only the mechanically educated know that the gas pedal controls an air valve, that gears do not move such that they could grind, and that engines cannot flood by pushing the gas pedal too much). From the current perspective, children are learning how to successfully interact in a social situation. Consequently, learning with respect to the box per se is tied to what children are learning about the social interaction that, as a whole, defines the situation.

\footnotetext{
${ }^{5}$ See Want and Harris (2001) for an example of a research design that tests for a causal learning interpretation for how to operate a novel artifact.
} 
In general, the relevant interactive affordances of an artifact/object are going to change depending on the type of situation that is involved (e.g., consider the different affordances between the legendary Wayne Gretzky's hockey stick in the hockey hall of fame and the "same" one sitting on the bench at a local iceskating rink). One methodological consequence of excluding this context-dependent nature for object affordances is that researchers have been overly focused on the "objective/causal" structure of the box independently of the social situation. However, what is relevant about an object depends on one's purposes and these will vary in different types of situations. Therefore, future research may benefit from incorporating the child's purpose/motivation for engaging with an object in a way that is tied to their understanding of the broader type of situation.

\section{Social-Motivational Perspective}

The results from hypothesis 3 provided evidence that motivational aspects are an essential variable for understanding why children over-imitate. However, fully recognizing the relevance of motivational aspects for over-imitation requires incorporating a broader appreciation for the heterogeneity of social situations in general. Knowledge about different types of social situations (and any corresponding motivations) must be learned and that forces a theoretical focus on children's developmental history of learning how to competently interact with other people in different types of situations.

One methodological consequence of excluding the broader social situation for the social-motivational perspective is that it has been overly focused on whether social agents are present or absent for some aspect of the experiment. While agents are certainly part of what makes a situation social in general, it is the commonly held understanding of how to engage with each other that makes it a social reality (Bickhard, 2008). Accordingly, this means that the presence of an agent may be most relevant for the learning of the social reality but is not essential for manifesting such knowledge once learned (e.g., other people need not be around to manifest the rituals of religion). Therefore, future research may benefit from focusing on the nature of these commonly held understandings and how they develop into types of social situations.

In sum, findings from the current study provided converging evidence that overimitation in preschoolers is less about the causal structure of the box and more about the social-cognitive structure of the situation as a whole (Kenward, Karlsson, \& Persson, 2011; Kiraly et al., 2013). This social-cognitive structure helps children to understand the purpose of the situation (motivational aspects) as well to define the meaning of the object in the situation (cognitive aspects). To put it simply, children are learning that "this is what you do to the artifact to participate in the social reality" rather than that "this is the necessary causal structure for how to open the box". 


\subsubsection{Re-thinking Novelty: Cognitive Aspects}

The results from hypotheses 1 and 2 suggest that children's existing knowledge about generic objects influences their presumptions about how a relatively novel box might operate? Whatever children (and adults) are able to learn through imitation, it must be within the context of what they already know about the world. That is, all imitation learning operates within the child's zone of proximal development. Imitation learning enables children to extend the boundaries of what they already know but does not enable learning something that is entirely novel. This may be both because of needing to know where to deploy attention when perceiving the demonstration but also because of needing to know enough about the domain to recreate the target actions. Learning to play sports by imitation highlights the limitations of both perceiving what actions the demonstrator provided and (unfortunately for aspiring sports stars) how to recreate them.

The results from hypothesis 2 (see Table 5.1) indicated differences in the total number of times that each of the 5 steps was reproduced at any point throughout the training phase. The step (step 4-tapping) that was least likely to be relevant for opening the box was produced the least followed by the other two, more likely, but also irrelevant steps (steps 1-button press \& 2-lever rotation), with the two relevant steps (steps 3-pin pull \& 5-pull knob) being imitated the most. We interpret this differential behavior as a consequence of children's zone of proximal development with respect to their knowledge about container like objects. That is, children with more robust object knowledge can better copy the less plausible target actions from the demonstration. In contrast, those children with less robust object knowledge may be more focused on learning about the box as physical object. This interpretation accords well with the developmental data from hypothesis 1 in which 3-years can be presumed to have less robust object knowledge than the 4- and 5-year-olds.

\subsubsection{Transcending Fidelity: Cognitive and Motivational Aspects}

The results of hypothesis 1 indicated that relevant target actions were imitated uniformly across the three age groups while there was a developmental increase in children's imitation of irrelevant target actions between 3- and 4-years of age (Fig. 5.1). Consider that age differences are acting as a surrogate for task difficulty and subsequent degree of uncertainty. Harnick (1978) demonstrated that imitation behavior in toddlers is influenced by task difficulty such that fidelity was greatest when the task was of moderate difficulty (i.e., not too difficult or too easy). Such a dynamic suggests that there are relevant motivational-learning processes involved with imitation learning (Kaplan \& Oudeyer, 2007). In the current task there is some amount of uncertainty for learning about both the object and the social reality involving the interaction with the adult. Therefore, the relative lack of over-imitation by the 3-year-olds suggests that they may have been motivated to learn more about the box than the broader social reality (about half did not over-imitate). In contrast, learning about the box may have been sufficiently trivial for the 4- and 5-year-olds 
such that they were motivated to learn about the more complicated social reality involving the box as a cultural artifact.

The suggested interpretation of these results does not propose an absolute claim about children of these ages engaging in a type of imitation (i.e., selective, over-, goal-, means-, social, cognitive, etc., Dickerson, Gerhardstein, Zack \& Barr, 2012; Over \& Carpenter, 2012). Instead, there is a task dependent dynamic that plays out for all imitation learning in which children imitate what they understand to be relevant about the demonstration and their determination about what is relevant depends on social-cognitive, cognitive, and motivational processes.

\subsubsection{Verbal Framing and Forgetting}

The results for hypothesis 4 indicated that the effect of verbal framing on children's imitation behavior was modest. Children in the problem-solving condition imitated with higher fidelity than those in the play condition; however, this difference only approached significance with respect to relevant target actions. Further, there was not the expected increase in imitation of irrelevant target actions for the play condition. Given some recent evidence for an effect of verbal framing on imitation fidelity (Herrmann et al., 2013), we believe that the subtlety of the effects in the current study are due to the weakness of the manipulation itself. In the current study: the transition from the warm-up period involved referring to the imitation task as the "boxes game" for both conditions; the task was presented with a playful and game-like structure; and, the objects involved were relatively unfamiliar (cf., Kiraly 2009; Herrmann et al. 2013). As for the manipulation, the only difference between conditions was whether the experimenter used the word "play" versus "open" when introducing the task (e.g., "Let me show you how to [play with/open] the boxes. I'm going to take two turns and then you'll get two turns"). Therefore, aspects common to both conditions contributed to the situation being about playing games and suggests that a stronger manipulation may find an effect.

\subsection{Conclusion}

The current study was meant to address two intimately related issues in the literature: First, how to reconcile the apparent conflict between selective imitation and automatic over-imitation? Second, how to account for why children overimitate? The analysis did not answer the first question directly but rather argued for an alternative characterization that highlights the selective nature of all forms of imitation activity. Consequently, the current study proceeded to explore the psychological basis for selectivity in a canonical over-imitation setting. In particular, how cognitive and motivational aspects were related to the broader type of social situation and how they collectively guided children's imitation activity. The results support our argument that children's over- and selective imitation depends on the 
specifics of the social situation. Specifically that is, we conclude that children overimitate because they are motivated to learn how to competently interact with people and objects in different types of social situations. That is, children over-imitate because it is the nature of learning about social realities.

What is special about imitation learning? Imitation is widely assumed to be a particularly important form of social learning because of its contribution to human culture more broadly. However, understanding of cultural artifacts does not reduce to the causally necessary ways of using objects or even to their instrumental functioning. Instead, cultural artifacts are saturated with aesthetics and their functioning is tied to our social interests as much as to our "rational" needs. Cultural artifacts are constrained by their physical embodiment but attain social meaning through social agents participating in social realities. Likewise, social ontology does not reduce to the presence or absence of social objects that are understood as more complex physical objects. Instead, social ontology is emergent from interactive systems grounded in the world. Social realities are fundamentally constrained by coordination and although they will involve arbitrary aspects (e.g., it does not matter what side of the road we drive on) that does not mean that there are no normative constraints for understanding what one ought to do to participate in cultural activities (i.e., rationality and necessity do not exhaust the normative ground for imitation learning).

The current action-based perspective offers a robust theoretical framework for exploring some of the richness of learning to participate in human culture. This framework draws on a model of the basic content of culture in terms of social ontology and provides a genuinely developmental perspective on those learning to participate in such activities. Standard frameworks in developmental psychology do not motivate consideration of how learning processes themselves must undergo learning and development. To the extent learning to learn is essential for the development of human forms of activity, it may be a productive avenue for roboticists to explore in order to realize the full potential of artificial systems. Further, a learning to learn framework highlights the need to consider the development of emergent forms of motivation, including social motivations, and helps explain why motivations for different forms of learning change with development (e.g., communicative imitation tends to decline around age 4 with the increased availability of learning through language, Nadel, 2006). Finally, standard frameworks define social ontology in terms of the ability to attribute mental-state contents to self and other (i.e., mindreading, theory of mind). However, the emergent origins of such mental-state contents (as well as the attribution process) remain a mystery (Carpendale \& Lewis, 2006). This does not mean that a mindreading framework toward social ontology cannot be implemented in robotics models (Dominey \& Warneken, 2010), but rather, that there are serious questions about whether that approach can adequately capture the nature, origins, and development of social robots (Thompson, Sameen, Maximilian, \& Racine, 2013).

In sum, the current empirical work and theoretical integration constitute a first step for a promising new avenue of research in the domain of social meta-learning 
that roboticists may well need to consider if they are to build social agents capable of participation in human socio-cultural activities.

Acknowledgements We would like to thank Mark Bickhard and Ulrich Müller for useful comments throughout writing the manuscript. We would also like to thank Andrea Lucas, Mitchel Harvey, and Bryan Hughes for help with data collection.

\section{References}

Allen, J. W. P., \& Bickhard, M. H. (2011a). Emergent constructivism. Child Development Perspectives, 5(3), 164-165.

Allen, J. W. P., \& Bickhard, M. H. (2011b). You can't get there from here: Foundationalism and development. Behavioral and Brain Sciences, 34, 124-125.

Allen, J. W. P., \& Bickhard, M. H. (2013a). Beyond principles and programs: An action framework for modeling development. Human Development, 56, 171-177.

Allen, J. W. P., \& Bickhard, M. H. (2013b). Stepping off the pendulum: Why only an action-based approach can transcend the nativist-empiricist debate. Cognitive Development, 28, 96-133.

Anisfeld, M. (2005). No compelling evidence to dispute Piaget's timetable of the development of representational imitation in infancy. In S. Hurley \& N. Chater (Eds.), Perspectives on imitation: From neuroscience to social science (Vol. 2, pp. 107-131). Cambridge: MIT Press.

Bauer, P. J., \& Fivush, R. (1992). Constructing event representations: Building on a foundation of variation and enabling relations. Cognitive Development, 7, 381-401.

Bickhard, M. H. (1980). Cognition, convention, and communication. New York: Praeger Publishers.

Bickhard, M. H. (2005). Functional scaffolding and self-scaffolding. New Ideas in Psychology, 23, $166-173$.

Bickhard, M. H. (2008). Social ontology as convention. Topoi, 27, 139-149.

Bickhard, M. H. (2009). The interactivist model. Synthese, 166, 547-591.

Bickhard, M. H. (in preparation). The whole person: Toward a naturalism of personsContributions to an ontological psychology. (In preparation)

Bickhard, M. H., \& Terveen, L. (1995). Foundational issues in artificial intelligence and cognitive science: Impasse and solution. Amsterdam/New York: Elsevier Scientific.

Buttelmann, D., Carpenter, M., \& Tomasello, M. (2009). Eighteen-month-old-infants show false belief understanding in an active helping paradigm. Cognition, 11, 337-342.

Call, J., \& Carpenter, M. (2002). Three sources of information in social learning. In K. Dautenhahn \& C. L. Nehaniv (Eds.), Imitation in animals and artifacts (pp. 211-228). Cambridge: MIT Press.

Call, J., \& Carpenter, M. (2009). Monkeys like mimics. Science, 325, 824-825.

Campbell, R. L., \& Bickhard, M. H. (1992). Type of constraints on development: An interactivist approach. Developmental Review, 12, 311-338.

Carpendale, J. I. M., \& Lewis, C. (2006). How children develop social understanding: Understanding children's worlds. Oxford: Blackwell.

Corriveau, K., \& Harris, P. L. (2009). Choosing your informant: Weighing familiarity and recent accuracy. Developmental Science, 12, 426-437.

Csibra, G., \& Gergely, G. (2009). Natural pedagogy. Trends in Cognitive Science, 13, 148-153.

Dickerson, K., Gerhardstein, P., Zack, E., \& Barr, R. (2012). Age-related changes in learning across early childhood: A new imitation task. Developmental Psychobiology, 55, 719-732.

Dominey, P. F., \& Warneken, F. (2010). The basis of shared intentions in human and robot cognition. New Ideas in Psychology, 29, 260-274. 
Gergely, G. (2003). The development of teleological versus mentalizing observational learning strategies in infancy. Bulletin of the Menninger Clinic, 67, 113-131.

Gergely, G., Bekkering, H., \& Kiraly, I. (2002). Rational imitation in preverbal infants: Babies may opt for a simpler way to turn on a light after watching an adult do it. Nature, 415, 755.

Gergely, G., \& Csibra, G. (2005). The social construction of the cultural mind: Imitative learning as a mechanism of human pedagogy. Interaction Studies, 6, 463-481.

Harnick, F. S. (1978). The relationship between ability level and task difficulty in producing imitation in infants. Child Development, 49, 209-212.

Harris, P. L., Corriveau, K., Pasquini, E. S., Koenig, M., Fusaro, M., \& Clément, F. (2012). Credulity and the development of selective trust in early childhood. In M. J. Beran, J. Brandl, J. Perner, \& J. Proust (Eds.), Foundations of metacognition (pp. 193-210). Oxford, England: Oxford University Press.

Herrmann, P. A., Legare, C. H., Harris, P. L., \& Whitehouse, H. (2013). Stick to the script: The effect of witnessing multiple actors on children's imitation. Cognition, 129, 536-543.

Heyes, C. (2005). Imitation by association. In S. Hurley \& N. Chater (Eds.), Perspectives on imitation: From neuroscience to social science (Vol. 1, pp. 157-176). Cambridge: MIT Press.

Horner, V., \& Whiten, A. (2005). Causal knowledge and imitation/emulation switching in chimpanzees (pan troglodytes) and children (homo sapiens). Animal Cognition, 8, 164-181.

Huang, C. T., Heyes, C., \& Charman, T. (2006). Preschoolers' behavioral reenactment of "failed attempts": The roles of intention-reading, emulation and mimicry. Cognitive Development, 21, $36-45$.

Jones, S. S. (2007). Imitation in infancy: The development of mimicry. Psychological Science, 18, 593-599.

Kaplan, F., \& Oudeyer, P. (2007). The progress drive hypothesis: An interpretation of early imitation. In K. Dautenhahn \& C. Nehaniv (Eds.), Models and mechanisms of imitation and social learning: Behavioral, social and communication dimensions (pp. 361-377). Cambridge: Cambridge University Press.

Kenward, B., Karlsson, M., \& Persson, J. (2011). Over-imitation is better explained by norm learning than by distorted causal learning. Proceedings of the Royal Society B, 278, 1239-1246.

Kiraly, I. (2009). The effect of the model's presence and of negative evidence on infants' selective imitation. Journal of Experimental Child Psychology, 102, 14-25.

Kiraly, I., Csibra, G., \& Gergely, G. (2013). Beyond rational imitation: Learning arbitrary means actions from communicative demonstrations. Journal of Experimental Child Psychology, 116, 471-486.

Lyons, D. E. (2009). The rational continuum of human imitation. In J. A. Pineda (Ed.), Mirror neuron systems: The role of mirroring processes in social cognition (pp. 77-103). New York: Humana Press.

Lyons, D. E., Damrosch, D. H., Lin, J. K., Deanna, M. M., \& Keil, F. C. (2011). The scope and limits of over-imitation in the transmission of artifact culture. Philosophical Transactions of the Royal Society B, 366, 1158-1167.

Lyons, D. E., Young, A. G., \& Keil, F. C. (2007). The hidden structure of overimitation. Proceedings of the National Academy of Sciences, 104(50), 19751-19756.

McCarthy, J., \& Hayes, P. (1969). Some philosophical problems from the standpoint of artificial intelligence. In B. Meltzer \& D. Michie (Eds.), Machine intelligence 4 (pp. 463-502). New York: American Elsevier.

McGuigan, N., Makinson, J., \& Whiten, A. (2011). From overimitation to super-copying: Adults imitate causally irrelevant aspects of tool use with higher fidelity than young children. British Journal of Psychology, 102, 1-18.

McGuigan, N., \& Whiten, A. (2009). Emulation and "overemulation" in the social learning of causally opaque versus causally transparent tool use by 23- and 30-month-olds. Journal of Experimental Child Psychology, 104, 367-381.

McGuigan, N., Whiten, A., Flynn, E., \& Horner, V. (2007). Imitation of causally opaque versus causally transparent tool use by 3- and 5-year-old children. Cognitive Development, 22, $353-$ 364. 
Meltzoff, A. N. (1995). Understanding the intentions of others: Re-enactment of intended acts by 18-month-old children. Developmental Psychology, 31, 838-850.

Nadel, J. (2006). Does imitation matter to children with autism? In S. J. Rogers \& J. H. G. Williams (Eds.), Imitation and the social mind: Autism and typical development (pp. 118-137). New York: Guilford Press.

Nehaniv, C. L., \& Dautenhahn, K. (2002). The correspondence problem. In K. Dautenhahn \& C. L. Nehaniv (Eds.), Imitation in animals and artifacts (pp. 41-62). Cambridge: MIT Press.

Nielsen, M. (2006). Copying actions and copying outcomes: Social learning through the second year. Developmental Psychology, 42, 555-565.

Nielsen, M., \& Blank, C. (2011). Imitation in young children: When who gets copied is more important that what gets copied. Developmental Psychology, 47, 1050-1053.

Nielsen, M., Simcock, G., \& Jenkins, L. (2008). The effect of social engagement on 24-montholds' imitation from live and televised models. Developmental Science, 11, 722-731.

Over, H., \& Carpenter, M. (2012). Putting the social into social learning: Explaining both selectivity and fidelity in children's copying behavior. Journal of Comparative Psychology, 126, 182-192.

Paulus, M., Hunnius, S., Vissers, M., \& Bekkering, H. (2011). Imitation in infancy: Rational or motor resonance? Child Development, 82, 1047-1057.

Piaget, J. (1954). The construction of reality in the child. New York: Basic.

Schulz, L. E., Hooppell, C., \& Jenkins, A. C. (2008). Judicious imitation: Children differentially imitate deterministically and probabilistically effective actions. Child Development, 79, 395410.

Southgate, V., Chevallier, C., \& Csibra, G. (2009). Sensitivity to communicative relevance tells young children what to imitate. Developmental Science, 12, 1013-1019.

Thompson, J. J., Sameen, N., Maximilian, B., \& Racine, T. P. (2013). Agnosticism gone awry: Why developmental robotics must commit to an understanding of embodiment and shared intentionality. New Ideas in Psychology, 31, 184-193.

Tomasello, M. (1996). Do apes ape? In C. M. Heyes \& G. Galef (Eds.), Social learning in animals: The roots of culture (pp. 319-346). San Diego, CA: Academic Press.

Uzgiris, I. C. (1981). Two functions of imitation during infancy. International Journal of Behavioral Development, 4, 1-12.

Want, S. C., \& Harris, P. L. (2001). Learning from other people's mistakes: Causal understanding in learning to use a tool. Child Development, 72, 431-443.

Whiten, A. (2006). The dissection of imitation and its "cognitive kin" in comparative and developmental psychology. In S. J. Rogers \& J. H. G. Williams (Eds.), Imitation and the social mind: Autism and typical development (pp. 277-309). New York: Guilford Press.

Whiten, A., McGuigan, N., Marshall-Pescini, S., \& Hopper, L. M. (2009). Emulation, imitation, over-imitation and the scope of culture for child and chimpanzee. Philosophical Transactions of the Royal Society B, 364, 2417-2428.

Williamson, R. A., \& Markman, E. M. (2006). Precision of imitation as a function of preschoolers' understanding of the goal of the demonstration. Developmental Psychology, 42, 723-731.

Zentall, T. R. (2006). Imitation: Definitions, evidence, and mechanisms. Animal Cognition, 9, 335353. 